\title{
PATRIMONIO LOCAL EN UN MUNDO GLOBAL: PROCESOS DE PATRIMONIALIZACIÓN CULTURAL EN CONTEXTOS LOCALES DE ANDALUCÍA Y EL PAÍS VASCO
}

\author{
LOCAL HERITAGE IN A GLOBAL WORLD: HERITAGIZATION OF \\ CULTURE IN ANDALUSIAN AND BASQUE LOCAL CONTEXTS
}

Enviado em 04 de setembro de 2015 Aceito em 20 de novembro de 2015 Iñaki Arrieta Urtizberea ${ }^{1}$

Elodia Hernández León ${ }^{2}$

Agustí Andreu Tomàs ${ }^{3}$

\begin{abstract}
Resumen: La patrimonialización cultural se trata de un proceso en el que determinados elementos (materiales e inmateriales) pasan a convertirse en bienes culturales porque simbolizan una identidad colectiva. Este proceso presenta una gran complejidad por diferentes motivos: referentes identitarios diversos, multiplicidad de agentes participantes, variabilidad de valores e intereses o incidencia de determinados procesos globales. Así, en este trabajo, presentamos los resultados de un estudio de carácter etnográfico sobre dos proyectos patrimoniales llevados a cabo en Aracena (Andalucía) y en Legazpi (País Vasco). Nuestro análisis pone de relieve cómo estas dos realidades locales diferentes viven en las mismas fechas, finales del siglo XX e inicios del siglo XXI, procesos similares de patrimonialización. Si, en un primer momento, esos proyectos son preferentemente identitarios; a partir de la década de los 90, comienza a imperar la idea de que el patrimonio cultural puede ser un activo importante en el desarrollo local, todo ello impulsado por la Unión Europea.
\end{abstract}

Palabras clave: Patrimonial cultural. Identidad. Desarrollo local.

Abstract: Heritagization of culture deals with the process of ascribing certain objects or knowhow with cultural value and significance given their importance as symbols reflecting a collective identity. This process has presented emerging challenges for a number of reasons, including the referencing to or the grouping

\footnotetext{
${ }_{1}^{1}$ Antropólogo, professor no Departamento de Filosofia de los Valores da Universidade do País Vasco. Atua nas áreas de museus, patrimônio cultural e políticas culturais. E-mail: i.arrieta@ehu.es

${ }^{2}$ Antropologa, professora da Universidade Pablo Olavide, Sevilha. Atua nas áreas de fronteiras, identidades socioculturais, patrimônio cultural, território e paisagem. E-mail: vrculturaycompromiso@upo.es

${ }^{3}$ Antropologo, professor do Departamento de Antropologia e Filosofia da Universitat Rovira i Virgili.
} 
of various identifies, the multiplicity of members participating in the processes, and the variability in the values and interests, or incidences of global processes. This paper presents the results of an ethnographic study of two heritage projects in Aracena (Andalusia) and in Legazpi (Basque Country). Our analysis highlights the way in which these two areas, each with its own local reality, experienced toward the end of the twentieth and early twenty-first centuries similar processes of heritagization. While each begun with a particular emphasis on the construction of local identity, the present study found that beginning in the 1990s, and as a result of the European Union, there was a shift toward the idea that cultural heritage can play an important role in local development.

Keywords: Cultural heritage. Identity. Local development.

\section{PRESENTACIÓN}

Si se quiere hacer una visita al municipio de Aracena (Andalucía), tres son los espacios patrimoniales que el turista ha de conocer: la Gruta de las Maravillas, el Castillo y el Museo del Jamón. Sin embargo, entre los tres espacios hay, al menos, dos diferencias sustanciales. Mientras la Gruta y el Castillo son destinos turísticos desde hace décadas en torno al patrimonio natural e histórico; el Museo, al contrario, es de reciente construcción, inaugurado a principios de este siglo, y se articula en base a la producción agroalimentaria y a los modos de vida locales. Esta infraestructura cultural se plantea como:

Un modelo de museo diferente al resto, un centro de interpretación capaz de servir de referencia y homenajear a la industria porcina y a todos aquellos hombres y mujeres anónimos que iniciaron una actividad que ha marcado la vida de toda una comarca. Este museo debía ser un escaparate de la cultura popular que atesoran los pueblos de nuestro Parque Natural, desconocida por muchos pese a la fama mundial de jamón ibérico.

Por aquellos mismos años, en el norte de la Península Ibérica se inicia también otro proceso de patrimonialización. En este caso, éste se articula en torno al hierro y a su producción, y se da en el municipio industrial de Legazpi (País Vasco). Este proceso se concreta con la apertura al público de la ferrería Mirandaola y la construcción del Museo del Hierro Vasco, teniendo como objetivo principal, aunque no el único, el atraer visitantes Según el entonces alcalde del municipio: 
Es un proyecto que trata de resaltar la naturaleza y el pasado de Legazpi ligados al hierro, desde antiguas ferrerías a las concepciones industriales de los años 60 y hacer de ello un eje de trabajo y atención prioritaria, sin olvidar otras acciones de promoción industrial.

Hasta aquí hemos presentado las recreaciones de dos procesos patrimoniales con los que esas poblaciones han construido sus escenarios de ofertas patrimoniales y turísticas para la atracción de visitantes. Esas estampas sugieren algunos de los aspectos significativos acerca de las cuestiones principales que vamos a abordar en este artículo a partir de dos estudios de caso. Dos proyectos patrimoniales que presentan sus especificidades, pero que, a su vez, muestran características comunes en tanto que son expresiones locales de los procesos globales de construcción de discursos del patrimonio cultural. Estos procesos promovidos y sostenidos, en nuestros casos de estudio por la Unión Europea principalmente, están estrechamente relacionados con el desarrollo de las políticas de reconversión económica en municipios del sur de Europa.

Se trata, por tanto, de un análisis de los procesos de patrimonialización de la cultura a través de dos proyectos locales ubicados en dos territorios de España. A partir de estos casos vamos a aproximarnos al estudio de las narrativas y de las acciones de patrimonialización acaecidas desde la década de los noventa del siglo pasado. Estos procesos apuntan a la coexistencia de diferentes criterios de selección de elementos patrimoniales locales y a la implicación de diversos agentes en la definición de dichos criterios. Así, "nuevos" patrimonios emergen y los "tradicionales" se resignifican y se reutilizan. El presente artículo sigue, de este modo, la línea de los trabajos de investigación centrados en los procesos de patrimonialización, señalada por Waterton and Smith (2010), según la cual dichos procesos se inscriben en contextos específicos que hace falta describir y analizar, teniendo en cuenta sus dimensiones culturales, sociales, políticas y económicas (RAUTEMBERG, 2003).

\section{EL PATRIMONIO COMO PROCESO: DIVERSIDAD EN LOS DISCURSOS}

\section{Y EN LOS AGENTES.}

Aunque el concepto de patrimonio cultural está lejos de estar claramente definido para toda la comunidad patrimonial (CHOAY, 2009), aquí nos sumamos a la interpretación de aquellos autores que lo vinculan con la identidad colectiva (PRATS, 1997; THROSBY, 2001; MCLEAN, 2006; POULOT, 2006; SCHEINER, 2006; ASHWORTH, GRAHAM; TUNBRIDGE, 2007; CHOAY, 2007; TURGEON, 2010; WATERTON; SMITH, 2010; DAVIS, 2011). De este modo, se trata de un proceso en el que determinados objetos (materiales e inmateriales) pasan a convertirse en bienes culturales porque simbolizan 
una identidad colectiva. Asimismo, al ser un proceso, nos alejamos de una visión estática del patrimonio, según la cual los bienes culturales preexisten, esperando a ser descubiertos (RAUTENBERG et al., 2000). En definitiva, se trata de un proceso de selección dinámico.

Sin embargo, somos conscientes de que esa conceptualización, que introduce la identidad en la centralidad de la noción de patrimonio, no le resta complejidad, antes al contrario, lleva aparejada la dificultad de adentrase en procesos sociopolíticos de construcción de discursos y narrativas identitarias que en absoluto son unívocos o unilaterales. En él intervienen diferentes agentes que tratan de legitimar sus versiones dentro del colectivo social al que pertenecen, en función de sus valores e intereses. Por tanto, los procesos patrimoniales son "campos de tensión" (BENDIX, 2009), "campos de batalla" (KIRSHENBLATT-GIMBLETT, 2001) o "una lucha en el presente" (CANDAU, 2006) cuyo objetivo consiste en apropiarse de los referentes identitarios del conjunto de una sociedad. Así, son procesos complejos multidimensionales y no necesariamente consensuados ya que pueden dar lugar a patrimonios opresivos respecto a algunos colectivos (CROOKE, 2007), patrimonios disonantes (TUNBRIDGE; ASHWORTH, 1996; GRAHAM, 2002; ASHWORTH; GRAHAM; TUNBRIDGE, 2007; PORIA; AVITAL; REICHEL, 2009), patrimonios indeseables (MCLEAN, 2006) o patrimonios incómodos (PRATS, 1997; MESA-BAINS, 2004). En definitiva, como sostienen autores como Halbwachs (1968), Lowenthal (1998), Prats (2005) o Ashworth, Graham and Tunbridge (2007) el patrimonio se va construyendo o destruyendo a partir de los intereses y valores culturales, sociales, políticos y económicos del presente.

La insistencia en la compresión del patrimonio cultural como un proceso multidimensional, que puede comenzar a transformarse desde sus inicios (DE VARINE; GELLEREAU, 2007), pone el foco en los valores que entran en el juego patrimonial: una línea de investigación e intervención que arranca con Aloïs Riegl (RAUTENBERG, 2008) y que muchos autores han desarrollado (LIPE, 1984; THROSB, 2001; MASON; TORRE, 2001; LOW, 2002; MASON, 2000; ENGLISH HERITAGE, 2008; FOJUT, 2009). De este modo, son los valores que poseemos, los que están en la base de una mirada selectiva que enfoca y hace que algunos elementos de la realidad "se vuelvan significativos para nosotros, y solo esos" (WEBER, 1973).

En el caso del patrimonio cultural, son aquellos valores culturales referidos a las identidades colectivas, los que legitiman la selección y el reconocimiento de unos elementos y no otros. No obstante, la centralidad de lo identitario en la construcción del sentido que adquieren los elementos patrimoniales en los diferentes contextos, no significa que sea lo único que haya que tener en cuenta en los procesos de valorización. 
Variables económicas, políticas, urbanísticas, educativas, profesionales, de prestigio emergen e impulsan las definiciones y las valorizaciones de lo que es patrimonio.

La pluralidad y las jerarquías de valores que entran en juego en el campo patrimonial nos devuelven al inicio de este apartado donde nos preguntábamos sobre los agentes que participan en la patrimonialización. De entre los agentes implicados en los procesos de patrimonialización en nuestras actuales sociedades democráticas merece especial atención la Administración pública. En particular, el estado nacional por ser uno de los actores principales en legitimar una determinada opción identitaria, creando y consolidando el discurso patrimonial autorizado (SMITH, 2011). Sin embargo, en la actualidad, a pesar de que los gobiernos nacionales desarrollen (legislen y ejecuten) las políticas culturales en el campo del patrimonio (declaraciones de bienes culturales, financiación de restauraciones y puestas en valor...) no son los únicos agentes político-administrativos que gobiernan los procesos de patrimonialización. Comparten liderazgo con organismos internacionales y gobiernos regionales y locales. En este sentido, el campo del patrimonio no es ajeno al actual contexto de globalización y localización (BORJA; CASTELLS, 1987; GIDDENS, 1990, p. 16; APPADURAI, 1995, p. 204; ORTIZ, 1997, p. 18; BECK, 1998, p. 103; BAUMAN, 2001; COHEN, 2001; OLIVA; CAMARERO, 2002; YÚDICE, 2002; GARCÍA CANCLINI, 2004)

Otros agentes importantes en esos procesos de patrimonialización son los especialistas y profesionales del patrimonio cultural. En muchos casos, son ellos los que objetivan el discurso patrimonial autorizado y las acciones patrimoniales dirigidas por el poder político (THROSBY, 1997). Se recurren a ellos como autoridad autorizada, como administradores del pasado.

No obstante, en los citados procesos también participan muchos mas agentes como son: los agentes económicos, las asociaciones, colectivos o eruditos locales o, simplemente, los ciudadanos.

Es aquí, en el estudio de esta relación de la articulación de los valores e intereses de los diferentes agentes en los procesos de patrimonialización, donde la perspectiva cualitativa y las técnicas etnográficas y la antropología aportan un valor añadido (LOW, 2002; BOUCHENAKI, 2006; BENDIX, 2009) a los estudios acerca del patrimonio cultural. Para describir y analizar cómo operan los agentes implicados en dichos procesos, consideramos un instrumento metodológico adecuado el concepto de campo definido por Bourdieu. Aunque este concepto haya sido abiertamente criticado (GARCíA CANCLINI, 2004, 2010; BENNETT et al., 2009), consideramos que es una herramienta metodológica válida puesto que nos permite poner el foco de atención en el conjunto de agentes que entran en juego en el campo patrimonial a partir delos valores, los intereses y el volumen y la composición de sus capitales - cultural, social, simbólico o 
económico - (ALVAREZ SOUSA, 1996; GARCÍA CANCLINI, 2004). Como sostiene el sociólogo francés, el campo es una relación de fuerzas entre los diferentes agentes e instituciones implicados con el objetivo de hacerse con el control (BOURDIEU, 2000). Desde esta perspectiva, analizaremos como se imponen unos discursos y acciones de activación de los patrimonios sobre otros en relación a las hegemonías y subalternidades. En palabras de Bourdieu (2000, p. 112-113):

Sabemos que en todo campo encontraremos una lucha, cuyas formas específicas hay que investigar en cada caso, entre el nuevo ingresado que trata de hacer saltar los cerrojos de la cuota de ingreso y el dominante que trata de defender el monopolio y de excluir la competencia.

\section{ARACENA Y LEGAZPI: LA ACTIVACIÓN DE LOS PATRIMONIOS}

\section{LOCALES}

Iniciábamos este artículo con dos escenas paralelas correspondientes a dos museos: el del jamón en Aracena y el del hierro, en Legazpi. Ambos museos son inaugurados en el 2005 y son de titularidad municipal. Aunque, como mostraremos más adelante, ambos proyectos tienen sus semejanzas, se dan en contextos locales significativamente diferenciados. Si bien Aracena y Legazpi se ubican en el mismo estado nacional, ambos municipios se inscriben en territorios políticos, socioeconómicos y culturales diferentes: el de Andalucía y el del País Vasco. El primero al sur de España, la segunda al norte.

Aracena está en el norte de Andalucía. Pertenece a la cadena montañosa de Sierra Morena, que dibuja el límite del territorio andaluz. La explotación de la dehesa ha sido históricamente su principal actividad económica, aunque también se pueden destacar el comercio y el contrabando, propiciados por su situación fronteriza con Portugal. La dehesa constituye un sistema de producción ancestral que se quiebra cuando en la década de los 60 del pasado siglo se inicia el proceso de desarrollo industrial en España, el denominado "desarrollismo franquista". En este proceso Andalucía juega el papel de proveedora de mano de obra a otros territorios industriales del Estado, como Cataluña y el País Vasco. Aracena pasa de tener unos 8.000 habitantes en 1950 a unos 6.500 a principios de los años 90 .Se produce un intenso éxodo rural hacia dichos polos industriales y una quiebra de los sistemas de producción tradicionales, al igual que otras poblaciones de la montaña española y del sur europeo.

A unos mil kilómetros al norte de Aracena, está Legazpi, en el interior del País Vasco. Este municipio ha tenido una vinculación estrecha con el hierro y su producción. 
Hasta el siglo XIX contaba con un número importante de ferrerías hidráulicas. Sin embargo, es con la construcción de la empresa Patricio Echeverría (PESA) a principios del siglo XX, cuando los trabajos relacionados con el hierro y el acero se vinculan estrechamente con la dicha localidad. Si a finales del XIX Legazpi es un municipio de unos 1.000 habitantes, dedicados fundamentalmente a la agricultura y la ganadería, en 1980 pasa a tener 11.000 personas. Este espectacular aumento demográfico se produce por PESA, en cuyas instalaciones trabaja prácticamente el total de la población activa del municipio. La actividad industrial trae cambios demográficos, económicos, sociales, culturales y urbanísticos significativos en el municipio. Toda la vida local gira en torno a dicha empresa. Pero toda esa actividad industrial comienza a entrar en crisis en la década de los 80 , como en otros muchos territorios europeos, perdiendo población y dinamismo económico.

La situación de crisis económica de los 80 coincide en España con un cambio importante en el campo de la política: el fin de la dictadura de Franco. El dictador fallece en 1975 y se inicia el periodo de transición hacia la democracia. Este cambio político va a traer un importante dinamismo social en el que expresiones políticas, culturales e identitarias, hasta entonces negadas, se van a reivindicar y expresar. De este modo, el estancamiento económico en el que aterriza la democracia española no impide la emergencia de un gran número de activaciones patrimoniales en todo el Estado a manos de asociaciones culturales, políticas y de la ciudadanía en general, como un modo de reafirmar su identidad local y cultural, hasta entonces negada y reprimida.

Asimismo, la Administración pública en España va a modificarse sustancialmente en ese periodo de cambios políticos. El poder político se va a descentralizar. Las diferentes regiones se constituyen en comunidades autónomas y van a asumir una serie de competencias políticas y administrativas, antes en manos del gobierno nacional, que les van a permitir gestionar, en cierta medida, sus territorios. Aunque es imposible desarrollar en este artículo la complejidad institucional de la administración pública en España, merece la pena subrayar que en el ámbito de la cultura las comunidades autónomas cuentan con importantes competencias. Esto les permite desarrollar sus propias políticas culturales, al margen de los criterios del Gobierno nacional. Igualmente, hay que destacar que, tras el fin de la dictadura, los ayuntamientos van a asumir muchas competencias y van a tener un gran protagonismo en todo aquello que tiene que ver con la gestión de su territorio. Entre ellas están las competencias en cultura y patrimonio.

En nuestros casos de estudio ese dinamismo cultural, social y político tiene su reflejo en el inicio de la puesta en valor del patrimonio cultural con el objetivo de refirmar y construir una comunidad territorial local, de orgullo y de pertenencia, que contrarresta con la situación de decadencia económica del momento. De hecho los 
discursos que denuncian el estado de abandono del patrimonio local y la necesidad de su recuperación aparecen junto a la reivindicación del apoyo de la administración pública para que palie los efectos de la situación de crisis económica.

En Aracena, desde inicios de los ochenta, comienzan a establecerse asociaciones en defensa del patrimonio local. Anualmente, desde1985, organizan en un municipio de la comarca de la sierra las jornadas sobre patrimonio cultural. Estas asociaciones municipales federadas tienen como objetivo el de velar por la conservación del patrimonio histórico, muy deteriorado. El discurso imperante de estas asociaciones civiles en los inicios era el de conservar y recuperar castillos, iglesias, ermitas y yacimientos: "es verdad que se tenía cierta obsesión por los temas del patrimonio histórico-artístico y arqueológico" (SANCHA SORIA, 2010). Para ello denunciaban la falta de subvenciones y de consideración de la administración autonómica respecto a la sierra y a su patrimonio. En esos años la valorización que se hace del patrimonio es claramente identitaria. Se trata de proteger algo que es propiedad de la población local y que la identifica.

En aquellas mismas fechas, en Legazpi, un grupo de vecinos interesados en la historia municipal y pertenecientes a una asociación cultural de reciente constitución, comienza a constituir el campo patrimonial local, vinculando Legazpi y su población local con la elaboración del hierro y el acero. Así, publican varias obras acerca de dicha vinculación histórica y actual, imparten conferencias y llevan a cabo diferentes trabajos de recuperación y puesta en valor de elementos, instalaciones y espacios industriales. Poco a poco todo ese trabajo se va sedimentando hasta convertirse "en una de las señas de identidad más importantes de Legazpi y aceptadas como tal por los legazpiarras", tal como lo expresa el Alcalde del municipio (AGUIRRE, 2005), y cuya expresión más nítida va a ser la propuesta de construcción de un museo del hierro. Así, el valor predominante en el inicio del proceso de patrimonialización del hierro es fundamentalmente el identitario.

De este modo tanto en Aracena como Legazpi, son principalmente los criterios identitarios los que articulan el discurso patrimonial autorizado. Un discurso iniciado e impulsado por algunos vecinos pertenecientes a asociaciones culturales locales que activan el valor los bienes culturales en la construcción de la identidad colectiva de cada municipio. Aunque los ayuntamientos se muestran interesados en ofrecerles apoyo, son ellos los principales agentes en los orígenes de esos procesos de patrimonialización. Las administraciones públicas supramunicipales no muestran mayor interés en esos procesos por ser patrimonios que adquieren valor y sentido a escala local. En aquellos años, tras el fin de la dictadura, las prioridades de los gobiernos autonómicos a la hora de consolidar las identidades colectivas, al margen, en mayor o menor grado, de la 
identidad nacional propuesta por el Gobierno del Estado, están en otros niveles de identidad y en otros ámbitos. En particular, en la conservación de grandes monumentos andaluces y vascos y en la creación de los medios de comunicación públicos y en el establecimiento de una red pública de educación. En el País Vasco, además, el Gobierno Vasco destina importantes recursos económicos y humanos a la recuperación de la lengua vasca, duramente reprimida durante la dictadura franquista.

Sin embargo, esa situación inicial de construcción de las identidades locales a través del patrimonio se va a ir transformando a lo largo de la década de los 90. Poco a poco otra valoración de tipo económico se va a ir imponiendo. Comienza a imperar la idea de que la cultura, en general, y el patrimonio cultural, en particular, pueden ser activos importantes en el desarrollo local (THROSBY, 2001; ANDREU, 2007). Así, según ese planteamiento los bienes y paisajes culturales pueden favorecer la diversificación económica, impulsado el sector de servicios, al convertirse en lugares de recreo y de consumo cultural para los turistas generando procesos de valorización patrimonial que Laplante (1992) denomina "sacralisation turistique". Procesos que encajan en el sistema económico de tipo postfordista que según Cuvelier, Torres y Gadrey (1994) incide en la aparición de un turismo "versátil" opuesto al clásico "turismo de masas". Esta vinculación entre los bienes culturales y el desarrollo económico implicará también la diversificación de los actores que participan en estos procesos (DE VARINE, 2005). Las nuevas estrategias patrimoniales serán impulsadas por la Unión Europea que como objetivos principales de sus programas y fondos estructurales (FERNÁNDEZ DE PAZ, 2006). No obstante, es una tendencia bastante generalizada a nivel mundial a medida que el "paradigma del economicismo cultural" (ZALLO 2011) se va extendiendo.

De este modo, el valor económico gana legitimidad decayendo los valores asociados al uso identitario que había dominado, como hemos visto, hasta la fecha. La centralidad del valor económico en la legitimación y puesta en valor del patrimonio cultural explica principalmente el empeño de construir un museo del jamón en Aracena. Como manifiesta un político local: "no creo que esto [el museo] se hiciera para conservar el patrimonio. Se hace para el turismo, esto es turismo y no patrimonio" (M.M. político municipal entrevistado el 06/07/2014).

Efectivamente, el proyecto de musealización de la actividad de producción y transformación del cerdo se lleva a cabo gracias a los fondos europeos Leader I, II y Plus, entre cuyos objetivos está financiar aquellos proyectos promovidos por los agentes locales y que se basen en la puesta en valor del patrimonio y de las producciones autóctonas. El fin de estos proyectos debe ser fomentar el desarrollo local y la diversificación económica. La creación preceptiva de grupos de desarrollo rural regentados por técnicos en cuya manos está el desarrollo e impulso de los proyectos 
europeos (HERNÁNDEZ, 2008), traerá a la primera línea del campo patrimonial a estos nuevos agentes de desarrollo local. Técnicos y políticos locales concentran los esfuerzos sobre el patrimonio en la creación de recursos turísticos. El campo patrimonial estará dominado por la activación del patrimonio de interés turístico, quedando las asociaciones ocupadas en el desarrollo de los estudios sobre los bienes patrimoniales y en ocasiones mostrando su disconformidad frente a las políticas locales que ponen el riesgo el patrimonio: "las asociaciones han tenido que ponerse al frente de movimientos sociales para impedir que se cometan agresiones al patrimonio, como fue el caso de las obras que pretendían hacer en la Ladera del Castillo de Aracena" (SANCHA SORIA, 2010).

En el caso de la construcción del Museo del jamón hay una estrategia clara: "retener a la gente en Aracena", ofreciéndoles un producto cultural más y, de este modo, prolongar su estancia y, por tanto, aumentar el consumo hostelero en el municipio (F.G. técnico en turismo entrevistado el 11/02/2014). Y no se han defraudado las expectativas. Se ha conseguido una ampliación en el tiempo medio de estancia del turista en la población. De hecho, desde su inauguración en 2005 , el museo ha ido incrementando anualmente sus visitas desde los 4.000 visitantes iniciales hasta los más de 23.000 ocho años después. Si tenemos en cuenta que los museos arqueológicos de ciudades tan importantes como Granada o Almería han recibido, en el mismo año del 2013, 815 o 11.000 visitas respectivamente, no cabe duda de que este museo municipal no ha sido un fracaso. Además, a nivel municipal es el segundo recurso turístico más importante, por detrás del de la Gruta de las Maravillas patrimonio de interés geológico-minero.

Pero el impacto económico del Museo del Jamón no está solamente en el número de visitantes que acoge y en el consumo hostelero que realizan. También lo tiene en la venta de productos del cerdo en el municipio, otro de los objetivos definidos en el proyecto inicial por parte del gobierno local de Aracena. A través del Museo se prestigian dichos productos, buscando que éstos sean más competitivos respecto a los de otros municipios próximos como los de Jabugo. Población ubicada a pocos kilómetros y que ha dado nombre al producto más preciado del cerdo: el jamón de pata negra y de bellota "Jamón de Jabugo".

Si en Aracena la valoración económica se va imponiendo a la identitaria, algo parecido ocurre en el municipio vasco de Legazpi. A lo largo de la década de los 90 del pasado siglo la relación entre el patrimonio cultural y el desarrollo local y el turismo se va a ir haciendo más estrecha. Así, aunque en el proyecto inicial del museo del hierro no se contemplaba apenas dicha relación, ésta comienza a afianzarse en el ámbito político. Por ejemplo, el Gobierno Vasco comienza a preparar planes para impulsar el turismo en el País Vasco y a organizar jornadas informativas acerca del papel que los museos y el patrimonio cultural pueden jugar en dicho impulso. Recordemos que estamos en un 
periodo de crisis industrial y que las instituciones públicas vascas buscan implementar nuevas actividades económicas en el territorio.

Junto a esa nueva legitimación política de carácter más general, la incorporación de una asesoría, solicitada por el Ayuntamiento, al proyecto del museo va a cambiar significativamente su orientación. Esta asesoría plantea la posibilidad de pedir una ayuda económica a la Unión Europea ya que, como ya hemos afirmado anteriormente, esa institución viene apoyando proyectos patrimoniales entre cuyos objetivos esté el desarrollo local a través del turismo.

Todo ello altera significante el campo patrimonial de Legazpi. En primer lugar, porque aquél grupo de vecinos, que iniciaron el proyecto por motivos fundamentalmente identitarios, son excluidos del desarrollo posterior de la iniciativa museística. $Y$, en segundo lugar, porque el turismo cultural se va a convertir en el principal eje articulador de dicho proyecto.

El espaldarazo definitivo a esa nueva orientación del proyecto viene de la Unión Europea cuando dicha institución concede una subvención del 50\%, a través del programa RESIDER II, para la construcción del museo. Sin embargo, este apoyo europeo va a plantear un reto importante al Ayuntamiento ya que éste tendrá que aportar un millón de euros al proyecto museístico en un periodo de crisis y de disminución de ingresos municipales. Para muchos políticos locales y vecinos esa inversión en el museo iba a conducir a un fuerte endeudamiento del Ayuntamiento y, consecuentemente, a un recorte de las subvenciones, de los gastos o de las inversiones en otros ámbitos. No obstante, el Alcalde decide seguir con el proyecto adelante, inaugurándose el museo en el 2005.

A lo largo de la primera década del presente siglo, el número visitantes al museo es de unos 20.000 al año. Un número importante si tenemos en cuenta que antes de su construcción la afluencia de visitantes al municipio era inexistente. No obstante, la exclusión de aquel grupo de vecinos del proyecto no va evitar que de vez en cuando emerja el conflicto en el campo patrimonial de Legazpi acerca de los significados y los usos dados al patrimonio local.

Aunque a finales de la década de los 80 y principios de los 90 la valorización identitaria de los bienes culturales locales es dominante, es claro que ambos museos, inaugurados en el 2005 , son resultado de un proceso de puesta en valor del patrimonio cultural en el que prima la valorización económica. En este periodo patrimonial un nuevo discurso patrimonial autorizado se establece, legitimado claramente por las instituciones públicas, desde las europeas hasta las locales, pasando por las regionales. 


\section{CONCLUSIONES}

A través de los ejemplos, a cuyo análisis nos hemos acercado sintéticamente, mostramos cómo dos realidades locales diferentes vivirán en las mismas fechas procesos similares de activación patrimonial. Si hasta hace poco las construcciones sociales del patrimonio estaban estrechamente relacionadas con la creación de identidades colectivas y con la recuperación de la memoria, en los últimos tiempos se ha consolidado una nueva mirada sobre el patrimonio que lo percibe como un elemento clave en el desarrollo económico y social de las comunidades locales. Desde esta perspectiva el patrimonio se convierte en un recurso económico, en un producto de consumo. El desarrollo de la sociedad capitalista ha creado nuevas necesidades de consumo basadas en el ocio y en la oferta turística que han provocado que los procesos de patrimonialización se inserten dentro de una perspectiva de rentabilidad económica y social.

Estos cambios en los usos del patrimonio, tanto en Aracena como en Legazpi, provocaran una modificación en las estrategias y del papel desarrollado por los diferentes agentes que intervienen en el campo patrimonial. Tras la generación de movimientos ciudadanos en torno a la valorización identitaria del patrimonio, con la aparición de los usos económicos el protagonismo pasará a los técnicos y especialistas. En ambos casos la participación activa de la población será subestimada y el discurso hegemónico sobre el patrimonio vendrá dictado por necesidades sociopolíticas y económicas controladas por los agentes vinculados a los gobiernos locales y puestas en práctica a través de la solvencia técnica de los especialistas del patrimonio que aflorarán en España fruto de las políticas públicas, tanto locales como regionales y europeas, que se fundamentan en la idea de que el patrimonio cultural se debe convertir en el eje del desarrollo local. De forma que se puede decir que un uso mercantilista del patrimonio desactiva la potencialidad que este tiene para el impulso de la participación social.

El recorrido de nuestros casos en paralelo también nos muestra cómo se acude a elementos singulares que construyen una identidad local. Si bien el uso identitario del patrimonio pierde vigencia frente al uso económico, lo paradójico es que no se renuncia a este primero puesto que es necesario recrear un territorio-escenario atractivo para un turista que consume estética pero también autenticidad y singularidad y, por lo tanto, necesita encontrar algo del lugar y sólo del lugar. Aunque, al final, las valoraciones económicas va tomado preeminencia, esto no quita de que el sustrato sea algo específico del lugar, algo que define lo local: lo identitario. A veces, sin embargo, estos procesos tan proyectados hacia la externalización de una imagen auténtica para los turistas, tienen el peligro de convertirlas en comunidades virtuales puramente estéticas 
"comunidades instantáneas para el consumo instantáneo" (BAUMAN, 2003) y no en comunidades reales que se apropian de su territorio.

\section{BIBLIOGRAFIA}

AGUIRRE, Juan. Legazpi: burdinezko bihotza/corazón de hierro. Legazpi: Legazpiko Udala, 2005.

ALVAREZ SOUSA, Antonio. El constructivismo estructuralista: la teoría de las clases sociales de Pierre Bourdieu. Revista Española de Investigaciones Sociológicas, n. 75, p. 145-172, 1996.

ANDREU, Agustí. Más allá del museo: las activaciones económicas del patrimonio: de los parques naturales a las fiestas temáticas. In: ARRIETA URTIZBEREA, Iñaki (Ed.) Patrimonios culturales y museos: más allá de la historia y del arte. Bilbao: Ed. de la Universidad del País Vasco, 2007, p.61-86.

APPADURAI, Arjun. The production of locality. In: FARDON, Richard (Ed.) Counterworks: managing the diversity of knowledge. London; New York: Routledge, 1995, p. 204-225. ASHWORTH, Gregory; GRAHAM, Brian; TUNBRIDGE, John. Pluralising pasts: heritage, identity and place in multicultural societies. London: Pluto Press, 2007.

BECK, Ulrich. ¿Qué es la globalización? Barcelona: Paidós, 1998.

BENDIX, Regina. Heritage between economy and politics: an assessment from the perspective of cultural anthropology. In: SMITH, Laurajane; AKAGAWA, Natsuko (Eds.) Intangible Heritage. London; New York: Routledge, 2009. p. 253-269.

BAUMAN, Zygmunt. La posmodernidad y sus descontentos. Madrid: Akal, 2001. . Comunidad: en busca de seguridad en un mundo hostil. Madrid: Siglo XXI, 2003.

BENNETT, Tony et al .Culture, class, distinction. Abingdon; New York: Routledge, 2009.

BORJA, Jordi; CASTELLS, Manuel. Local y global: la gestión de las ciudades en la era de la información. Madrid: Taurus; United Nations for Human Settlements, 1997.

BOUCHENAKI, Mounir. Editorial. Museum international: patrimonio inmaterial, n. 221-222, p. 7-12, 2006.

BOURDIEU, Pierre. Cuestiones de sociología. Madrid: Istmo, 2000.

CANDAU, Joël. Antropología de la memoria. Buenos Aires: Nueva Visión, 2006. 
CHOAY, Françoise. Alegoría del patrimonio. Barcelona: Gustavo Gili, 2007.

. Le patrimoine en questions: anthologie pour un combat. Paris: Seuil, 2009.

COHEN, Elie. Globalización y diversidad cultural. In: Informe mundial sobre la cultura: 20002001. Paris: UNESCO; Mundi-Prensa, 2001, p. 66-91.

CROOKE, Elizabeth. Museums and community: ideas, issues and challenges. London; New York: Routledge, 2007.

CUVELIER, Pascal; TORRES, Emmanuel; Gadrey Jean (ed.). Patrimoine, modèles de tourisme et développement local. Paris: L’Harmattan, 1994.

DAVIS, Peter. Ecomuseums: a sense of place. London; New York: Continuum, 2001.

DE VARINE, Hugues. Les Racines du futur: le patrimoine au service du développement local. Lusigny-sur-Ouche: Asdic, 2005.

DE VARINE, Hugues; GELLEREAU, Michèle. Accueil, publics... discuter les choix implicites: pour un engagement des acteurs de la scène patrimoniale. Culture et musées, n.10, p.134-138, 2007.

ENGLISH Heritage. Conservation principles: policies and guidance. London: English Heritage, 2008.

FERNÁNDEZ DE PAZ, Esther. De tesoro ilustrado a recurso turístico: el cambiante significado del patrimonio cultural. Pasos: Revista de Turismo y Patrimonio Cultural, v.4, n.1, p. 1-12, 2006.

FOJUT, Noel. Les origines philosophiques, politiques et pratiques de la convention. In: Le patrimoine et au-delà. Strasbourg: Editions du Conseil de l’Europe, 2009, p. 15-24.

GARCÍA CANCLINI, Néstor. Diferentes, desiguales y desconectados. Barcelona: Gedisa, 2004.

GIDDENS, Anthony. Consecuencias de la modernidad. Madrid: Alianza, 1990.

GREFFE, Xavier. ¿Es el patrimonio un incentivo para el desarrollo? Boletín del Instituto Andaluz del Patrimonio Histórico, n. 42, p. 43-51, 2003.

GRAHAM, Brian. Heritage as knowledge: capital or culture? Urban Studies, v.39, n. 5-6, p. 10031017, 2002.

HALBWACHS, Maurice. La mémoire collective. Paris: Presses Universitaires de France, 1968. 
HERNÁNDEZ LEÓN, E. De parques naturales a parques urbanos. turismo y patrimonialización del territorio en áreas protegidas. In: BELTRAN, Oriol; PASCUAL, José; VACCARO, Ismael (Ed.) Patrimonialización de la naturaleza: el marco social de las políticas ambientales. San Sebastián: Ankulegui, 2008, p. 83-96.

KIRSHENBLATT-GIMBLETT, Barbara. La cultura de les destinacions: teoritzar el patrimoni. Revista d'Etnologia de Catalunya, n. 19, p. 44-61, 2001.

LAPLANTE, Marc. Le patrimoine en tant qu'attraction touristique: histoire, possibilités et limites. In: NEURET, Rédis (Ed.). Le patrimoine, atout du développement. Lyon: Presses Universitaires de Lyon, 1992, p. 49-61.

LIPE, William D. Value and meaning in cultural resources. In: CLEERE, H. F. (Ed.). Approachesto the archaeological heritage. Cambridge: Cambridge University Press, 1984, p. 1-11.

LOULANSKI, Tolina; LOULANSKI, Vesselin. The sustainable integration of cultural heritage and tourism: a meta-study. Journal of Sustainable Tourism, v. 19, n. 7, p. 837-862, 2011.

LOW, Setha M. Anthropological-ethnographic methods for the assessment of cultural values in heritage conservation. In: DE LA TORRE, Marta (Ed.). Assessing the values of cultural heritage. Los Angeles: The Getty Conservation Institute, 2002, p. 31-49.

LOWENTHAL, David. El pasado es un país extraño. Madrid: Akal, 1988

MASON, Randall. Assessing the values of cultural heritage. In: DE LA TORRE, Marta (Ed.). Assessing the values of cultural heritage. Los Angeles: The Getty Conservation Institute, 2002, p. 5-30.

MASON, Randall; DE LA TORRE, Marta. Valores y conservación del patrimonio en las sociedades en proceso de globalización. In: Informe mundial sobre la cultura: 2000-2001. Paris: UNESCO; Mundi-Prensa, 2001, p. 164-179.

MCLEAN, Fiona. Introduction: heritage and identity. International Journal of Heritage Studies, v. 12, n. 1, p. 3-7, 2006.

MESA-BAINS, Amalia. The real multiculturalism: a struggle for authority and power. In: ANDERSON, Gail (Ed.) Reinventing the museum: historical and contemporary perspectives on the paradigm shift. Lanham: Altamira, 2004, p. 99-109.

PORIA, Yaniv; BIRAN, Avital; REICHEL, Arie. Visitors' preferences for interpretation at heritage sites. Journal of Travel Research, v. 48, n.1, p. 92-105, 2009.

POULOT, Dominique. Introduction. Culture et Musées, n.8, p. 13-25, 2006. 
PRATS, Llorenç. Antropología y patrimonio. Barcelona: Ariel, 1997.

. Concepto y gestión del patrimonio local. Cuadernos de Antropología Social, n. 21, p.

17-35, 2005.

RAUTENBERG, Michel. Comment s'inventent de nouveaux patrimoines: usages sociaux, pratiques institutionnelles et politiques publiques en Savoie. Culture et Musées, n.1, p. 19-43, 2003.

. Introduction du patrimoine comme œuvre au patrimoine comme image. In: NEMERY Jean-Claude; RAUTENBERG; Michel; THURIOT, Fabrice (Ed.) Stratégies identitaires de conservation et de valorisation du patrimoine. Paris: L'Harmattan, 2008, p. 9-18.

RAUTENBERG, Michel et al. Introduction. In: Campagnes de tous nos désirs: patrimoines et nouveaux usages sociaux. Paris: Maison des Sciences de l’Homme, 2000, p.1-10.

OLIVA, Jesús; CAMARERO, Luis. Paisajes sociales y metáforas del lugar: una exploración de la ruralidad en Navarra. Pamplona: Universidad Pública de Navarra/Nafarroako Unibertsitate Publikoa, 2002.

ORTIZ, Renato. Mundialización y cultura. Madrid: Alianza, 1997.

SANCHA SORIA, Felix. El patrimonio serrano: conocimiento, conservación y difusión en 25 años de jornadas de patrimonio. Conferencia dictada durante las $\mathbf{X X}$ Jornadas del Patrimonio de la Sierra. Cumbres Mayores (Huelva) 12 de Abril 2010.

SCHEINER, Tereza Cristina. Museología e interpretación de la realidad: el discurso de la historia. In: Museología e historia: un campo del conocimiento. Córdoba (Argentina): ICOFOM, 2006, p. 60-67.

SMITH, Laurajane. El 'espejo patrimonial': ¿̇llusión narcisista o reflexiones múltiples? Antípoda, v.12, p. 39-63, 2011.

THROSBY, David. Making preservation happen: the pros and cons of regulation. In: SCHUSTER, J.; MONCHAUX, J. de; RILEY, C. (Eds). Preserving the built heritage. Hanover: University Press of New England, 1997, p. 32-48.

. Economía y cultura. Madrid: Cambridge University Press, 2011.

TUNBRIDGE, John; ASHWORTH. Gregory. Dissonant heritage: the management of the past as a resource in conflict. Chichester: John Wiley and Sons, 1996. 
TURGEON, Laurier. Du matériel à l'immatériel : nouveaux défis, nouveaux enjeux. Ethnologie Française, v. 40, n. 3, p. 389-399, 2010.

WATERTON, Emma; SMITH, Laurajane. The recognition and misrecognition of community heritage. International Journal of Heritage Studies, v. 16, n. 1, p. 4-15, 2010.

WEBER, Max. Ensayo sobre metodología sociológica. Buenos Aires: Amorrortu, 1973.

YÚDICE, George. El recurso de la cultura: usos de la cultura en la era global. Barcelona: Gedisa, 2002.

ZALLO, Ramón. Industrias culturales y territorios creativos: los límites de la transversalidad. In: BUSTAMANTE, Enrique (Ed.). Las industrias creativas: amenazas sobre la cultura digital. Barcelona: Gedisa, 2011. p. 153-190. 